\title{
Comparación del uso de anestésico local versus inyección de lidocaína bajo guía ecográfica para el control del dolor en pacientes sometidos a biopsia de próstata
}

\author{
Medina Márquez C, Cadena González Y, Guerra Garzón A, Pérez Hidalgo JM.
}

Fundación Cardio Infantil. Bogotá. Colombia.

Actas Urol Esp. 2006;30(10):987-990

\section{RESUMEN}

COMPARACIÓN DEL USO DE ANESTÉSICO LOCAL VERSUS INYECCIÓN DE LIDOCAÍNA BAJO GUÍA

ECOGRÁFICA PARA EL CONTROL DEL DOLOR EN PACIENTES SOMETIDOS A BIOPSIA DE PRÓSTATA

Propósito: Evaluar el efecto de la aplicación de lidocaína gel versus la colocación periprostática de lidocaína en el control del dolor en pacientes sometidos a biopsia de próstata.

Materiales y métodos: Se incluyeron los pacientes que ingresaron a la FCI-IC para la realización de biopsia de próstata bajo guía ecográfica. Se dividieron los pacientes aleatoriamente en dos grupos de 22 pacientes cada uno con características similares, a uno de los cuales se le realizó la biopsia de próstata previa colocación de 10 cc de lidocaína gel intrarrectal y al otro grupo se le suministró de 10 cc de lidocaína al 1\% en los ángulos vésico-prostáticos bajo guía ecográfica. Para la evaluación del dolor se utilizó la escala análoga visual de dolor durante y después del procedimiento en ambos grupos. El procedimiento rutinario de toma de biopsias por octantes y la preparación de los mismos fue el mismo y no cambia en ningún caso.

Resultados: La media de dolor durante el procedimiento fue de 2,0 para el grupo de anestésico inyectado y 4,77 para el grupo de gel. Después del procedimiento, la media de dolor fue de 0,77 y 3,14 respectivamente. Complicaciones como bacteremia se presentó en $3(6,8 \%)$ pacientes del total, los cuales estaban en el grupo de gel y ninguna en el grupo de anestesia inyectada. No se encontró relación significativa con otras variables.

Conclusiones: La aplicación de lidocaína periprostática es eficaz en el control del dolor en pacientes sometidos a biopsia de próstata, además es un procedimiento seguro y fácilmente reproducible en nuestro medio.

Palabras clave: Biopsia. Anestesia. Próstata.

\section{ABSTRACT}

COMPARISON OF THE USE OF LOCAL ANAESTHETIC VERSUS LIDOCAINA'S INJECTION UNDER ULTRASOUND GUIDE FOR THE PAIN CONTROL IN PATIENTS LED TO PROSTATE BIOPSY

Objective: To evaluate the lidocaine gel's application effect versus the periprostatic placement of lidocaine to manage the pain in patients who go through a prostate biopsy.

Materials and Method: We took the patients who entered the FCI-IC to effectuate a prostate biopsy with an echographic guideline. The patients were split in two groups of 22 people with each one bearing similar characteristics. One of these groups experimented the previous prostate biopsy with 10cc of intrarectal lidocaine gel and the other group experimented $10 \mathrm{cc}$ of lidocaine to $1 \%$ in the vesic-prostatic through echographic guidelines. To evaluate the pain, we used the visual analogue scale to gauge the pain during and after the procedure in both groups. The daily procedure to do biopsies by octants and their subsequent preparation remained the same and never changed.

Results: The average scale of pain during the procedure was 2.0 for the group with injected anaesthesia and 4.77 for the group who used gel. After the procedure the average of pain was 0.77 and 3.14 respectively. Some complications as bacteremy were present in 3 patients $(6.8 \%)$ of the total, who were in the gel group and none were found in the group of injected anestesia. No significant relation was found with respect to other variables.

Conclusions: the application of periprostatic lidocaine is efficient to control the pain in patients who go through a prostate biopsy. Besides, it is a safe procedure which can be easily reproduce in our environment. 
$\mathrm{E}^{1}$ cáncer de próstata es el segundo cáncer más frecuente en el hombre ${ }^{1}$, diversos métodos se realizan en busca de llegar al diagnóstico de cáncer de próstata, como son el tacto rectal y el antígeno prostático específico ${ }^{2}$. En todos los casos el diagnóstico definitivo se hace con la biopsia de próstata. Diversos estudios, han mostrado diferentes métodos de realizar la biopsia de próstata con el fin de disminuir los niveles de dolor a los cuales se someten los pacientes durante la realización del procedimiento $^{3-5}$.

Tradicionalmente, la biopsia prostática se realiza colocando lidocaína gel como lubricante previo al procedimiento, con un escaso control del dolor ${ }^{6}$; se han estudiado algunos métodos nuevos como es la colocación de otras formas de anestesia, ya sea mediante medicamentos ingeridos vía oral o colocación intraprostática de anestésico en forma inyectada ${ }^{7-9}$.

Uno de los objetivos principales del estudio, consiste en demostrar la eficacia de la aplicación de lidocaína inyectada, en los ángulos vésico prostáticos bajo guía ecográfica para el control del dolor, en pacientes sometidos a biopsia de próstata; asimismo demostrar que es un procedimiento seguro, sencillo y eficaz para el control del dolor en estos pacientes ${ }^{10,11}$ que en algunas ocasiones se ven abocados a la realización de un mayor número de biopsias o a la realización de procedimientos adicionales ${ }^{12,13}$.

\section{MATERIAL Y MÉTODOS}

Es un estudio de intervención, prospectivo, aleatorizado en el cual se incluyeron los pacientes que ingresaron a la FCI-IC para la realización de biopsia de próstata bajo guía ecográfica. Los pacientes deben tener indicación de realizar la biopsia de próstata y no cumplir con ninguno de los criterios de exclusión. La totalidad de los pacientes tiene consentimiento informado para la realización del procedimiento.

Los pacientes se dividieron en 2 grupos de 22 integrantes cada uno, de forma aleatoria la cual se llevó a cabo mediante el programa RALLOC utilizando bloques de variables de tamaño 2 y 4 , para la realización del procedimiento. Al primer grupo se le colocó previo a la realización de toma de muestras para biopsia una dosis de $5 \mathrm{ml}$ de lidocaína al $1 \%$ en los ángulos vésico prostáticos bilateralmente bajo guía ecográfica, al segundo grupo de pacientes se le aplicó 10 cc de lidocaína gel al $2 \%$ intrarrectalmente previo al procedimiento, posteriormente se realizó la toma de muestras por octantes en condiciones iguales para los dos grupos examinados.

Una vez el procedimiento finaliza se le preguntó al paciente acerca del grado de dolor y molestia con el procedimiento usando la escala visual análoga de dolor la cual va de 0 (Cero, ausencia total de dolor) a 10 (Diez, peor dolor jamás experimentado por el paciente) puntos. Veinte minutos posterior al procedimiento se reinterrogó al paciente sobre el grado de dolor que se experimentó con el procedimiento.

Se evaluó la tolerancia al procedimiento con cada uno de los métodos y se evaluó si hay o no diferencia significativa en el grado de dolor en cada uno de los grupos estudiados.

Se correlacionaron otras variables como son la edad y el grado de dolor con cada uno de los métodos; asimismo los niveles de PSA, el volumen prostático por ecografia, los hallazgos ecográficos sugestivos de malignidad y la realización o no de biopsias previas.

Los pacientes fueron vistos posteriormente y se evaluó el índice de seguridad realizando medición de posibles complicaciones y encontrar si hay diferencias significativas entre los dos grupos estudiados.

\section{RESULTADOS}

Un total de 44 pacientes ingresaron al estudio, los cuales fueron divididos en 2 grupos cada uno de 22 integrantes, uno del grupo de anestésico inyectado y otro grupo con gel. Demográficamente los 2 grupos fueron estadísticamente iguales. La edad promedio fue de 62 años en el grupo de anestesia inyectada y de 59 años en el grupo de gel; asimismo no se presentaron diferencias significativas ni en el valor de PSA, ni en el volumen de la próstata por ecografia, ni en la indicación del procedimiento (Tabla 1).

A los pacientes se les preguntó la percepción del dolor durante el procedimiento y 20 minutos después del mismo usando la escala análoga del dolor. Se anotaron las diferencias entre los dos 
Tabla 1

Características iniciales de los pacientes

\begin{tabular}{|c|c|c|c|c|c|c|}
\hline \multirow{3}{*}{$\begin{array}{l}\text { Anestesia } \\
\text { Pacientes }\end{array}$} & \multicolumn{3}{|c|}{ Inyectada } & \multicolumn{3}{|c|}{ Gel } \\
\hline & \multicolumn{3}{|c|}{22} & \multicolumn{3}{|c|}{22} \\
\hline & Mínimo & Máximo & Promedio & Mínimo & Máximo & Promedio \\
\hline Edad & 42 & 77 & 62,23 & 47 & 72 & 59,32 \\
\hline PSA & 1,6 & 33,4 & 10,98 & 3,5 & 57,1 & 10,0 \\
\hline Volumen cc & 14 & 110 & 46,32 & 20 & 100 & 44,5 \\
\hline
\end{tabular}

grupos. En el grupo de anestesia inyectada el mínimo dolor durante el procedimiento fue de cero y el máximo de 5 con una media de 2,0, al realizarles la misma pregunta 20 minutos posteriores acerca de la percepción del dolor durante el procedimiento la mínima fue cero y la máxima de 3 con una media de 0,77 .

Al grupo de gel, el mínimo dolor durante el procedimiento fue de 1 y el máximo de 9 con una media de 4,77 y después del procedimiento el mínimo fue de cero y el máximo de 6 con una media de 3,14. En ambos casos se aprecia una diferencia estadísticamente significativa (Tabla 2).

Se presentaron complicaciones en $3(6,8 \%)$ pacientes, dadas en todos los casos por bacteremias posterior a la biopsia de próstata, las cuales se resolvieron en su totalidad con manejo médico. Los tres pacientes en que se presentó bacteremia posterior a la biopsia fueron del grupo de gel y ninguna complicación en los pacientes con anestesia inyectada.

\section{DISCUSIÓN}

El uso de la anestesia inyectada se ha venido utilizando últimamente en la realización de biopsia de próstata. Durante el estudio, se tomaron 2 grupos de características similares a los cuales se les aplicó, a uno de ellos anestesia en forma inyectada, en cada uno de los ángulos vésico prostáticos, bajo guía ecográfica y el otro grupo se realizó de la manera tradicional con lidocaína gel. No se apreciaron diferencias en cuanto a volumen de próstata, edad, nivel de PSA y el número de biopsias previas en la percepción del dolor.

Después de la colocación de anestesia inyectada este grupo presentó una menor percepción del dolor durante y después del procedimiento, siendo estadísticamente significativa respecto al grupo de gel. El procedimiento se realiza de una manera sencilla y es fácilmente reproducible. Durante la aplicación del anestésico los pacientes no muestran mayor molestia y no retrasa la realización del mismo.

La realización del procedimiento se realizó de la misma manera en ambos casos. El grupo de anestesia inyectada, no presentó ningún tipo de complicación relacionada con la aplicación del anestésico, ni con el procedimiento en sí y en el grupo de gel 3 pacientes presentaron bacteremia posterior a la biopsia, las cuales resolvieron con tratamiento médico.

Se logra apreciar que la aplicación de anestesia inyectada bajo guía ecográfica, es un procedimiento seguro y eficaz para la realización de biopsias de próstata. Además, aporta una mayor tranquilidad al paciente y tolerancia al mismo, permitiendo la realización de un procedimiento más cómodo y en muchos casos permite la toma de un mayor número de biopsias cuando así se requiera.

Tabla 2

Comparación del dolor en los dos grupos

(Cero, ausencia total de dolor y diez, peor dolor jamás experimentado por el paciente)

\begin{tabular}{lcccccc}
\hline $\begin{array}{l}\text { Anestesia } \\
\text { Pacientes }\end{array}$ & \multicolumn{3}{c}{$\begin{array}{c}\text { Inyectada } \\
\text { Mínimo }\end{array}$} & 22 & & \multicolumn{2}{c}{ Gel } \\
\hline & Máximo & Media & Mínimo & Máximo & Media \\
Dolor Durante & 0 & 5 & 2,0 & 1 & 9 & 4,77 \\
Dolor Posterior & 0 & 3 & 0,77 & 0 & 6 & 3,14 \\
\hline
\end{tabular}




\section{CONCLUSIONES}

La aplicación de anestesia inyectada es un procedimiento eficaz en el control del dolor en pacientes sometidos a biopsia de próstata.

El uso de anestesia inyectada es un procedimiento sencillo y seguro para el control del dolor en pacientes llevados a biopsia de próstata.

No hay relación existente entre el control del dolor y los niveles de PSA, la edad, el volumen prostático y la realización de biopsias previas.

\section{Agradecimientos}

Fundación Cardio Infantil, Universidad El Bosque.

\section{REFERENCIAS}

1. Siroky M, Edelstein T, Krane R. Manual of Urology. 1999; Second Edition. Lippincott Williams and Wilkins.

2. Walsh P, Retik A, Vaughan D, Wein A. CAMPBELL'S Urology. 2003; Eighth Edition. Associate Editors.

3. Ovek C, Tunc B, Yalcin V, Solok V. Comparison of 3 different methods of anesthesia before transrectal prostate biopsy: a prospective randomized trial. Journal of Urology. 2004; 172(2):502-505.

4. Elliott L, Dundas D, Patel U. Randomized trial of lidocaine VS lidocaine/bupivacaine periprostatic injection on longitudinal pain scores alter prostate biopsy. Journal of Urology. 2004;171(1):247-250.

5. Mallick S, Humbert M, Braud F. Local anesthesia before transrectal ultrasound guided prostate biopsy: comparison of 2 methods in a prospective, randomized clinical trial. Journal of Urology. 2004;171(2):730-733.

6. Cevik I, Ozveri H, Akdas A. Lack of effect of intrarectal lidocaine for pain control during transrectal prostate biopsy: A randomized prospective study. European Urology. 2002;42 (3):217-220.
7. Inal G, Yazici S, Adsan O, Ozturk B, Kosan M, Cetinkaya M. Effect of periprostatic nerve blockade before transrectal ultrasound-guided prostate biopsy on patient comfort: A randomized placebo controlled study. International Journal of Urology. 2004;1 1(3): 148-151.

8. Rabets J, Jones J, Patel A, Zippe CD. Bupivacaine provides rapid, effective periprostatic anaesthesia for transrectal prostate biopsy. BJU International. 2004;93(9):12161217.

9. Peters J, Thompson A, Mcnicholas T, Hines JE, Hanbury DC, Boustead GB. Increased patient satisfaction from transrectal ultrasonography and biopsy Ander sedation. BJU International. 2001;87(9):827-830.

10. Obek C, Onal B, Ozkan B, Onder AU, Yalcin V, Solok V. Is Periprostatic Local Anestesia For Transrectal Ultrasound Guided Prostate Biopsy Associated with increased infectious or hemorrhagic complications? A prospective randomized trial. Journal of Urology. 2002;168(2):558-561.

11. Seymour H, Perry MJ, Lee-Elliot C, Dundas D, Patel U. Pain alter transrectal ultrasonography-guided prostate biopsy: the advantages of periprostatic local anaesthesia. BJU International. 2001;88(6):540-544.

12. Matlaga B, Lovato J, Hall Mc. Randomized prospective trial of a novel local anesthetic technique for extensive prostate biopsy. Urology. 2003;61(5):972-976.

13. Jones Js, Oder Mehmet, Zippe Craig. Saturation prostate biopsy with periprostatic block can be performed in the office. Journal of Urology. 2002;168(5):2108-2110.

\section{Dr. Yair Cadena González}

yaircadena@yahoo.com

(Trabajo recibido el 24 de julio 2006) 